\title{
Design and Validation of Dynamic Positioning for Marine Systems: A Case Study
}

\author{
Silvia Donnarumma, Massimo Figari, Michele Martelli, Stefano Vignolo, and Michele Viviani
}

\begin{abstract}
The design of a dynamic positioning (DP) system is a challenging task with several technical fields involved in the problem solution. Numerical simulation is a powerful tool to aid the designer during the system development and to speed up the design process. This paper presents the simulation methodology adopted to design and test the DP system for a vessel with a standard propulsion configuration. Simulation results and sea trial measurements are compared to illustrate the reliability of the proposed simulation platform.
\end{abstract}

Index Terms-Dynamic positioning, environmental disturbances, propulsion modeling, simulation-based design.

\section{INTRODUCTION}

A DYNAMICALLY positioned vessel has to maintain its position and heading (fixed location or predetermined track) exclusively by means of active thrusters. Dynamic positioning (DP) has come a long way in 70 years since its early applications, developing alongside the oil industry. An historical perspective of DP systems can be found in [5], [18]. Despite the present crisis of the oil industry, the research in this field is still ongoing, in view of the variety of DP applications. An exhaustive insight of the major technology advances in DP controller design having taken place during more than 30 years of research and development is reported in [17].

When dealing with particular operational requirements, as the DP ones, the prediction of the vessel performance via simulation procedures is a crucial aspect of the design process. The main advantages of mathematical and numerical simulation are the possibility of comparing different choices for control and allocation logic as well as the possibility to test the system in any (simulated) condition, also extreme, without any danger for people and vessel. Moreover, virtual testing of the system allows us to reduce sea trials and therefore costs and delivery times [2] as well as enhancing safety and reliability of the vessel [16].

Usually the DP control system is designed for vessels specifically suited for DP applications, where the number of the avail-

Manuscript received July 5, 2016; revised November 4, 2016 and April 12, 2017; accepted July 11, 2017. (Corresponding author: Silvia Donnarumma.) Associate Editor: K. Takagi.

S. Donnarumma and S. Vignolo are with the Department of Mechanical, Energy, Management and Transportation Engineering-DIME, University of Genoa, Genoa 16145, Italy (e-mail: donnarumma@dime.unige.it; vignolo@ dime.unige.it).

M. Figari, M. Martelli, and M. Viviani are with the Department of Naval Architecture, Electric, Electronic, and Telecommunication Engineering-DITEN, University of Genoa, Genoa 16145, Italy (e-mail: massimo.figari@unige.it; michele.martelli@unige.it; michele.viviani@unige.it).

Digital Object Identifier 10.1109/JOE.2017.2732298 able thrusters is highly redundant to control position and orientation of the vessel. In the present paper, the authors address the problem of designing a DP control system for a patrol vessel originally not designed for DP missions. The vessel propulsion control system, optimized for the navigation condition, has been required to provide, as a retrofit, a certain DP performance under moderate weather conditions. The plant layout, arranged with a combined diesel or electric propulsion system with two propellers and one bow thruster (BT), does not allow consideration of the problem as an underactuated control problem in the sense of [14]. Nevertheless, it had to face two main challenges. First, the overall architecture and hierarchy of the DP control system had to comply with the industrial standards of the company acting as DP and automation provider; this aspect introduced several constraints to the control logic development. Second was the development of a specific thrust allocation logic (TAL) tailored for the propulsion system, uncommon in DP applications.

For such a system, the adopted design strategy deeply involved simulation techniques since its preliminary phases. Indeed, a first simplified model was implemented to design the regulator logics [1]. Second, a thorough model of the vessel was developed to validate the controller [4] and to strengthen a useful structure for both real-time hardware in the loop (RTHIL) and sea trials prediction. To this aim, the simulation-based control design procedure developed by the authors for naval vessels [2], [11] has been adopted. The procedure presents some common aspects to those proposed by other authors [7], [17], but also some important differences. In particular, the procedure includes a full nonlinear propulsion and steering model able to represent the behavior of the ship plants with great detail. This latter aspect is not always properly addressed in the literature with possible drawbacks in simulation fidelity.

The proposed model-based design methodology consists of two separate phases. In the first one, the simulation platform is developed into two mutually interacting parts: the vessel and the controller. In the second step the RT-HIL is used to test the functional capability of the DP controller before its installation on board. The RT-HIL testing allows us to detect hidden software errors, erroneous configuration parameters, and design flaws [2], [12]. Moreover, the adoption of RT-HIL enables highlighting of some important aspects such as the programmable logic controller (PLC) inner computational time, possible transmission line delays, and the correct PLC discrete managing of continuous dynamical processes [8], [9].

The mathematical description of a dynamically positioned vessel includes distinct models for low- and wave-frequency 




Fig. 1. Simulation framework structure.

ship motions, slowly varying wind, current, and wave forces [7], [16]. As previously mentioned, a detailed propulsion and steering model is a fundamental aspect to ensure reliability of simulation results and, in particular, to implement more realistically the RT-HIL procedure. Accordingly, the two prime movers, the shaft line dynamics, the propellers, and the control surfaces are thoroughly modeled, with their mutual interactions and all the existing mechanical constraints.

For what concerns the DP controller, a three-independentaxes-proportional-derivative (PD) controller is implemented and coupled to an estimation of the mean environmental disturbances action, which replaces the integrative term. In detail, the different submodels included in the DP regulator are the error computation, the controller, the environmental forces compensation, the force allocation logic (FAL), and the TAL.

The resulting outcome is a DP simulation platform that can turn out to be a useful tool for controller designers. The detailed frame of the simulator is drawn in Fig. 1.

The layout of the paper is as follows. Section II describes the mathematical model of the vessel; it deals with the lowand high-frequency motion models and the propulsion plant modeling. Section III illustrates environmental force models. Section IV contains the detailed description of the DP control logic implemented in the simulator and on board. Finally Section $\mathrm{V}$ presents a comparison between simulation results and sea trials measurements. Section VI is devoted to conclusions.

\section{Vessel Mathematical Modeling}

The patrol vessel endowed with the DP system under study is about $90 \mathrm{~m}$ long and $10 \mathrm{~m}$ of breadth. The vessel is equipped with two uncoupled controllable pitch propellers (CPPs), two uncoupled flap rudders, and one BT. In DP maneuvers, this layout does not provide for redundancy: The failure of a single component jeopardizes the performance of the whole system.

The DP system is composed by one DP control console; the control station, located on the bridge; two portable stations available in the bridge for port maneuvering; and one more located

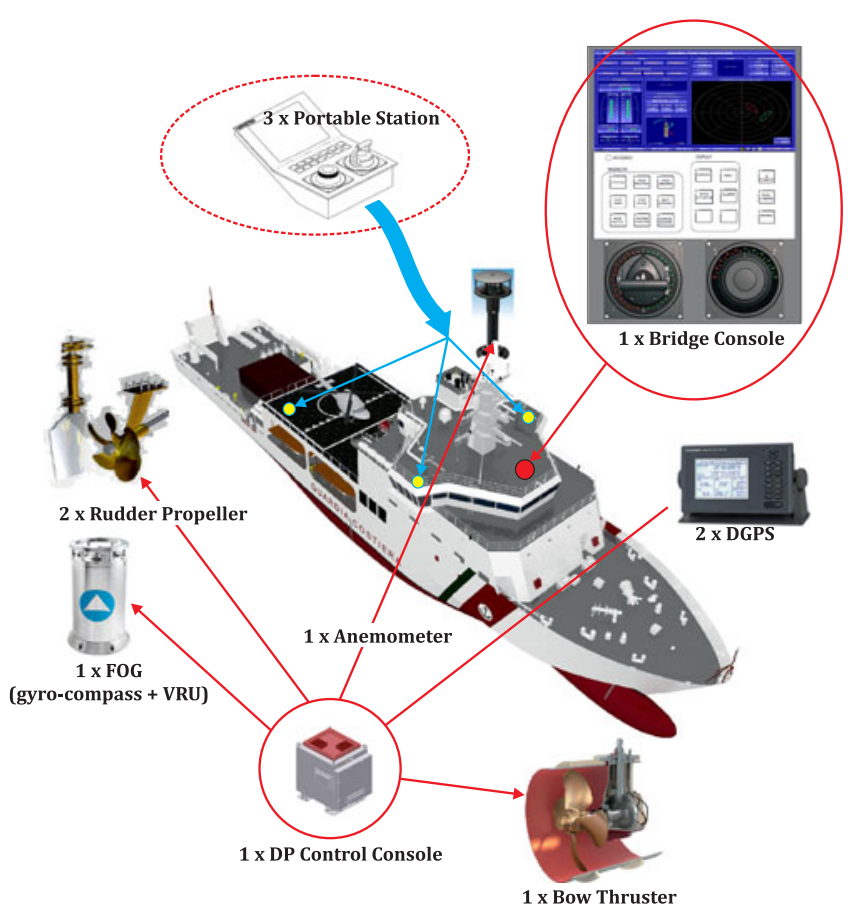

Fig. 2. DP system.

in the engine control room. The reference system comprises one fiber-optic gyro-compass (FOG), one vertical reference unit (VRU), the anemometer, and two differential GPS, as drawn in Fig. 2.

Two mathematical models are developed for the physical system and the control logic, of course interacting with each other. The controller outputs become the actuators setpoints and the vessel model output becomes the controller feedback in a closed loop.

The motion of the vessel is represented as the superposition of wave-frequency (WF) and low-frequency (LF) components. The latter are related to wave drift forces and wind action, which tend to move the vessel away from the required position, while the WF components represent an oscillatory motion around such a configuration.

\section{A. LF Motions}

When dealing with maneuvering problems, it is useful to introduce two reference frames, according to Fig. 3: the Earthfixed reference frame $\left\{\Omega, \underline{n}_{1}, \underline{n}_{2}, \underline{n}_{3}\right\}$ and the body-fixed frame $\left\{\mathcal{O}, \underline{b}_{1}, \underline{b}_{2}, \underline{b}_{3}\right\}$. The origin $\mathcal{O}$ is located on the mean water-free surface at midship. Adopting standard notations [6], kinematical and dynamical equations are given by

$$
\left\{\begin{array}{l}
\dot{x}=u \cos \psi-v \sin \psi \\
\dot{y}=u \sin \psi+v \cos \psi \\
\dot{\psi}=r
\end{array}\right.
$$

and

$$
\left\{\begin{array}{l}
m\left(\dot{u}-x_{G} r^{2}-u v\right)=X \\
m\left(\dot{v}+x_{G} \dot{r}+u r\right)=Y \\
I_{z} \dot{r}+m x_{G}(\dot{v}+r u)=N
\end{array}\right.
$$




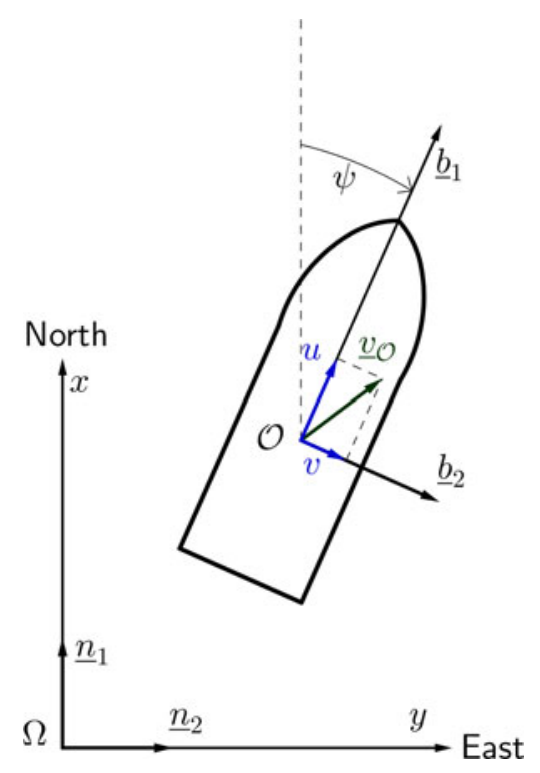

Fig. 3. Reference frames: Earth-fixed and body-fixed frames.

where $\underline{v}_{\mathcal{O}}=\dot{x} \underline{n}_{1}+\dot{y} \underline{n}_{2}$ and $\underline{v}_{\mathcal{O}}=u \underline{b}_{1}+v \underline{b}_{2}$ denote the linear velocity of $\mathcal{O}$ expressed in the $\underline{n}$-basis, and $\underline{b}$-basis, respectively, $\underline{\omega}=r \underline{b}_{3}$ indicates the angular velocity, $x_{G}$ represents the longitudinal coordinate of gravity center w.r.t. $\left\{\mathcal{O}, \underline{b}_{1}, \underline{b}_{2}, \underline{b}_{3}\right\}, m$ is the vessel mass, $I_{z}$ is the moment of inertia about $\underline{b}_{3}$-axis passing through $\mathcal{O}$, and $R=X \underline{b}_{1}+Y \underline{b}_{2}$ and $M=N \underline{b}_{3}$ are the force and the moment expressed in the $\underline{b}$-basis, respectively. In particular, $X=X_{h}+X_{p}+X_{e}, Y=Y_{h}+Y_{p}+Y_{e}+Y_{b}$, and $N=N_{h}+N_{p}+N_{e}+N_{b}$ and subscripts $h, p, e, b$, refer to hull, propellers, environmental, and BT forces and moments, respectively.

The hull forces and moments are implemented in accordance with [13]. They are thought of as the sum of main contributions due to ideal fluid, hull lifting, and cross-flow effects. Such contributions are indicated respectively by the subscripts $I, H L$, and $H C$, i.e.,

$$
\begin{gathered}
X_{h}=X_{I}+X_{H L}-R_{T} \\
Y_{h}=Y_{I}+Y_{H L}+Y_{H C} \\
N_{h}=N_{I}+N_{H L}-N_{H C}
\end{gathered}
$$

where $R_{T}$ represents the hull resistance measured through model tests. All different force and moment terms are computed according to [13], coupling drift and yaw motions. The input of the model is the relative velocity of the vessel with respect to the current: $u_{r}=u-u_{c}$ and $v_{r}=v-v_{c}$, where $u_{c}$ and $v_{c}$ are the longitudinal and lateral current velocity components, respectively. For sake of shortness, the complete formulations are not reported here; they can be found in [13].

\section{B. WF Motions}

Dynamically positioned vessels are subjected to irregular wave action. Such action can be split into two main components: the so-called first-order forces and moments, linearly proportional to the wave height, and the so-called second-order forces and moments proportional to the square of the wave height. The first-order forces and moments are large amplitude disturbances with zero-mean values and WFs. For DP applications, the compensation of first-order wave induced motions is, in general, not of interest, mainly because of their highfrequency content whose variation is too fast to be compensated by the actuators. However, WF motions can cause instabilities in the controller. For such a reason, WF motions are modeled separately and then added to the LF motions; LF motion components are then singled out by means of a cascade of two second-order Butterworth low-pass filters and sent to the regulator.

Borrowing from [3] and [15], WF motions are modeled as

$$
\begin{aligned}
& \dot{u}_{w f}+\omega_{p} u_{w f}=K_{x} \xi \\
& \dot{v}_{w f}+\omega_{p} v_{w f}=K_{y} \xi \\
& \dot{\psi}_{w f}+\omega_{p} \psi_{w f}=K_{\psi} \dot{\xi}
\end{aligned}
$$

where the triplet $\left\{K_{x}, K_{y}, K_{\psi}\right\}$ is defined as

$$
\begin{aligned}
& K_{x}=\chi_{x} \cos \gamma_{s} \\
& K_{y}=\chi_{y} \sin \gamma_{s} \\
& K_{\psi}=\chi_{\psi} \sin 2 \gamma_{s}
\end{aligned}
$$

$\left\{\chi_{x}, \chi_{y}, \chi_{\psi}\right\}$ accounts for the ship response amplitude operators (RAOs) for surge, sway, and yaw, respectively; $\gamma_{s}:=\psi_{s}-\psi$, $\psi_{s}$ is the main wave incoming angle, as shown in Fig. 6, $\omega_{p}$ is the sea peak frequency, and $\xi$ is the wave elevation time history. WF longitudinal and lateral velocities can be computed by solving (4a) and (4b); longitudinal and lateral motions can be computed by integrating $u_{\mathrm{wf}}$ and $v_{\mathrm{wf}}$, respectively. Finally, from (4c), yaw motion can be computed and yaw rate can be carried out by differentiating the result. The components of the surge, sway, and yaw motions in response to the sea action are equivalent to that of a suitable harmonic oscillator. Similarly, the sea action can be modeled by means of a mass-spring-damper system whose forces is a Gaussian white noise $W$; see [1].

The sea spectrum is modeled according to

$$
\ddot{\xi}+2 N \omega_{p} \dot{\xi}+\omega_{p}^{2} \xi=K_{J} \dot{W}
$$

where $\omega_{p}=2 \pi / T_{p}$ and $T_{p}$ is the mean period, $K_{J}=$ $K_{J}\left(H_{s}, T_{p}\right)$ is the coefficient that models the corresponding spectrum, $H_{s}$ is the significant wave height, and $N$ is a damping factor.

By moving from the time to the frequency domain and computing the corresponding spectra, a comparison between the approximated spectra and the original one is made possible. The result is shown in Fig. 4, where spectra are plotted for some sea states: from SS2 to SS5. Vessel RAOs approximation is shown in Fig. 5. High values of RAOs at low nondimensional frequency are visible, but this condition is far away from the standard domain of WF motions.

\section{Ship Propulsion Plant}

In this section, the model of the propulsion plant is presented. The resulting model yields the required thrusts necessary to 


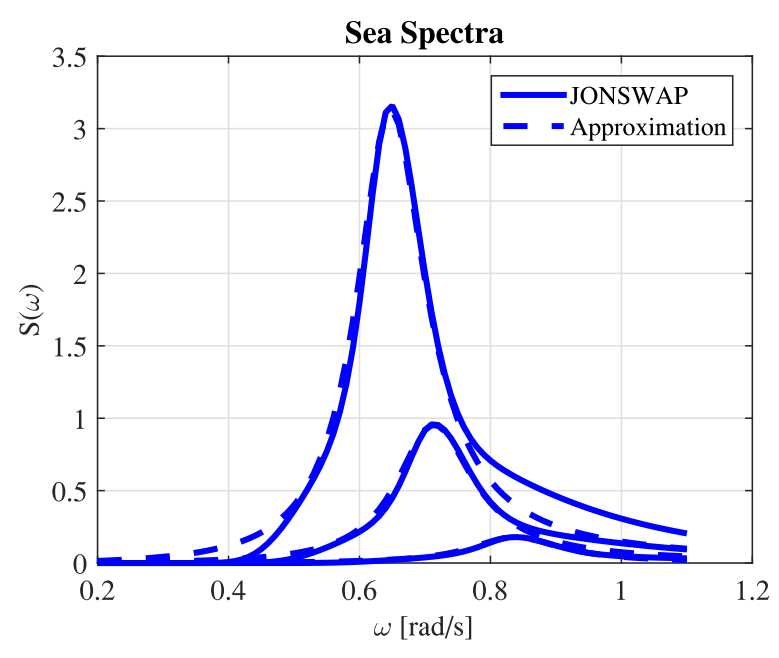

Fig. 4. Sea spectra approximation for some sea state.

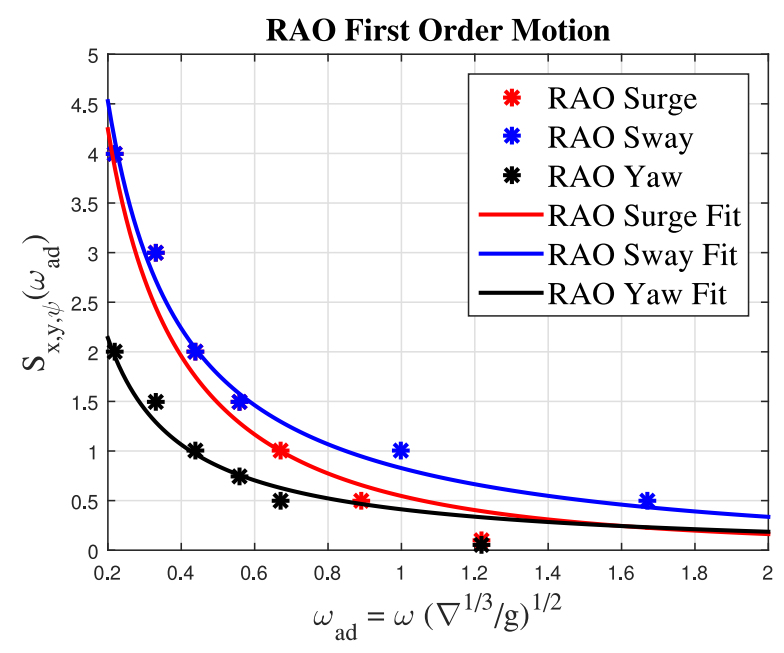

Fig. 5. Ship RAOs fit.

overtake environmental disturbances. The propulsion plant is composed by the following elements: main engines and their governors, the gearbox, the thrust and other bearings, the shaft line, and the two CPP with related rudders. A large number of variables are required to describe the propulsion plant dynamics, including ship speed, revolutions per minute, propeller pitch angle, and fuel supply. In a modern propulsion plant, all these variables are managed by the propulsion control system. In this case, the latter is bypassed by the DP controller.

The transmission line studied in this work has two degrees of freedom: the shaft line revolution regime and the propeller pitch angle. With respect to traditional transmission lines, this gives a greater operational flexibility. The dynamics of the propeller pitch depend on the load acting on the propeller blades and the dynamics of the pitch actuating mechanism [10].

The engine governor is modeled through the first-order dynamic equation

$$
\dot{m}_{f}^{R}=K_{P} e_{\mathrm{RPM}}+\int_{0}^{t} K_{I} e_{\mathrm{RPM}}(\xi) \mathrm{d} \xi
$$

where $\dot{m}_{f}^{R}$ is the fuel mass rate, $K_{P}$ and $K_{I}$ are the proportional and integral coefficient of the engine controller, respectively, and the shaft speed error $e_{\mathrm{RPM}}$ is defined as

$$
e_{\mathrm{RPM}}=\frac{100}{n_{\max }}\left(n_{R}-n_{D}\right)
$$

in terms of a percent of the difference between the setpoint $n_{R}$ and the actual shaft speed $n_{D}$, and $n_{\max }$ is the maximum rotation speed.

The introduction of saturation

$$
\dot{m}_{f}= \begin{cases}3, & \text { if } \dot{m}_{f}^{R}<3 \\ \dot{m}_{f}^{R}, & \text { if } 3<\dot{m}_{f}^{R}<100 \\ 100, & \text { otherwise }\end{cases}
$$

where the $3 \%$ is the self-reliance fuel mass, is needed to avoid undeliverable values. However, this can be a source of undesirable controller behaviors.

Once the actual fuel mass rate $\dot{m}_{f}$ is evaluated and the actual shaft speed is known, it is possible, through a surface interpolation, to obtain the engine delivered torque $Q_{\text {eng }}$. The lowest time-consuming method to evaluate the engine performance is the use of the response surface, often based on manufacturer data. The surface depends on the engine speed and fuel consumption flow rate.

The propeller hydrodynamic forces are evaluated through a quasi-steady methodology based on the open water propeller characteristics, by which it is possible to evaluate the thrust coefficient $K_{T}$ and torque coefficient $K_{Q}$. For CPP, these coefficients depend on the blade pitch angle $\varphi$ and on the advance ratio

$$
J=\frac{V_{a}}{n D}
$$

where the advance speed $V_{a}$ is the axial speed relative to the undisturbed fluid, $D$ is the propeller diameter, and $n$ is the shaft line revolution expressed in revolutions per second.

It is then possible to calculate the hydrodynamic force along the $\underline{b}_{1}$ direction, the thrust $T$, and the required propeller torque $Q_{P}$, using the following formulas:

$$
\begin{aligned}
T & =K_{T}(J, \varphi) \rho n^{2} D^{4} \\
Q_{P} & =K_{Q}(J, \varphi) \rho n^{2} D^{5}
\end{aligned}
$$

where $\rho$ is the sea water mass density.

The dynamics of each shaft line is described by

$$
\frac{\mathrm{d} n}{\mathrm{~d} t}=\frac{1}{I}\left(Q_{\mathrm{eng}}-Q_{p}-Q_{\text {fric }}\right)
$$

where $Q_{\mathrm{eng}}$ is the engine, $Q_{\text {fric }}$ friction, and $Q_{P}$ propeller torques, and $I$ the total axial inertia of the engine/gear/shaft/propeller system. In the case of a twin screw ship, where the two shaft lines can be used uncoupled or locked together, strong asymmetries, in terms of shaft loads, can be experienced during tight maneuvers.

The rudder forces are evaluated through a quasi-staticmethodology, using lift and drag coefficients $C_{L}$ and $C_{D}$. In addition, the whole interaction between rudder and propeller is taken into account following the approach described in [11]. 


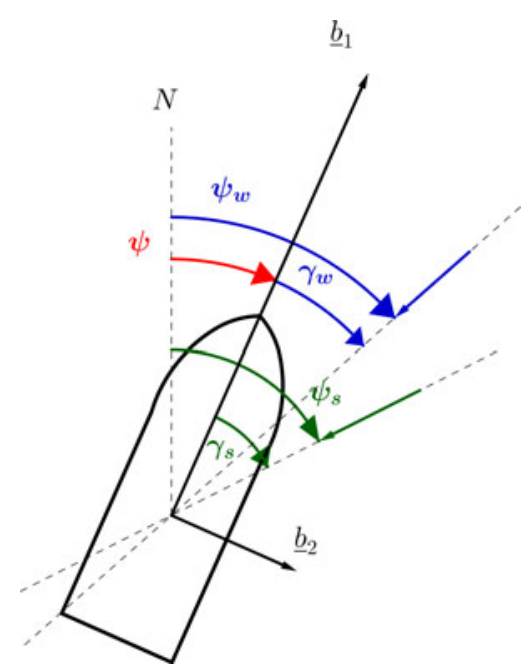

Fig. 6. Relative incoming disturbance directions: The blue lines refer to wind, while the green ones refer to sea.

\section{DISTURBANCES}

Environmental forces and moments are modeled by means of the linear superposition of wave and wind actions, according to

$$
\begin{aligned}
X_{e} & =X_{\text {wind }}+X_{\text {waves }} \\
Y_{e} & =Y_{\text {wind }}+Y_{\text {waves }} \\
N_{e} & =N_{\text {wind }}+N_{\text {waves }} .
\end{aligned}
$$

\section{A. Waves Modeling}

Second-order forces are characterized by nonzero mean values and low frequencies. Because they tend to force the vessel in a slow drift motion, they are called wave-drift forces and moments; see [15]. Such forces and moments can be considered to be proportional to the square of the wave height $H$. For this case of study, JONSWAP spectrum has been chosen and modeled, as shown in Section II-B. The square of the wave height time history is computed as the square of the wave elevation time history envelope, carried out from the spectrum. Such envelope is computed through the square root of the signal itself summed with same the signal shifted out of phase by $\pi / 2$. The resulting expressions for the force and moment are

$$
\begin{aligned}
X_{\text {waves }} & =H^{2} C_{x_{s}} \cos \gamma_{s} \\
Y_{\text {waves }} & =H^{2} C_{y_{s}} \sin \gamma_{s} \\
N_{\text {waves }} & =H^{2} C_{n_{s}} \sin 2 \gamma_{s}
\end{aligned}
$$

where $C_{x_{s}}, C_{y_{s}}$, and $C_{n_{s}}$ are the dimensional coefficients of the wave drift force and moment computed for head sea, lateral sea, and bow quartering sea, respectively; $\gamma_{s}:=\psi_{s}-\psi$ is the relative angle between the main wave incoming direction and the vessel bow, as shown in Fig. 6.
CONTROL SYSTEM MODULAR STRUCTURE

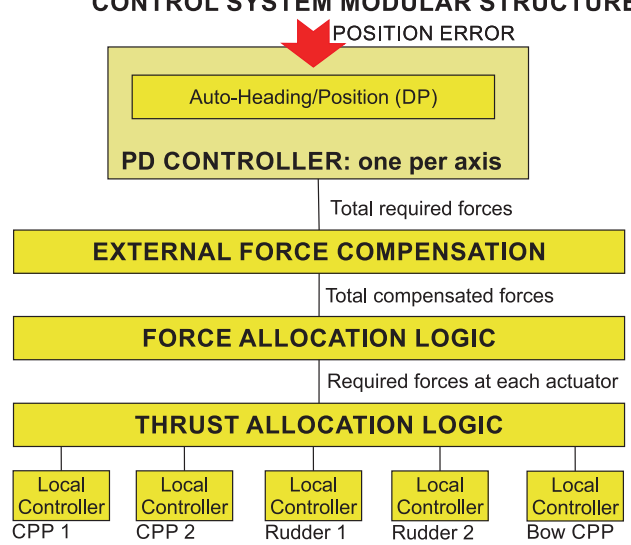

Fig. 7. DP regulator modular structure.

\section{B. Wind Modeling}

Wind action is computed as the linear superposition of the mean force and moment due to wind mean speed $V_{R}$ and the effects due to gusts speed $v_{g}$. The Davenport spectrum is approximated to generate gusts time history as it has been done for sea spectrum in Section II-B. Once wind speed time history is carried out, force and moment are computed as proportional to the square of the wind speed and suitable coefficients depending on the main direction of the apparent wind and on the exposed area

$$
\begin{aligned}
X_{\text {wind }} & =\frac{1}{2} \rho_{a} V_{a w}^{2} A_{f t} C_{x_{w}} \cos \gamma_{a w} \\
Y_{\text {wind }} & =\frac{1}{2} \rho_{a} V_{a w}^{2} A_{l t} C_{y_{w}} \sin \gamma_{a w} \\
N_{\text {wind }} & =\frac{1}{2} \rho_{a} V_{a w}^{2} A_{l t} L C_{n_{w}} \sin 2 \gamma_{a w}
\end{aligned}
$$

where $\rho_{a}$ is the air mass density; $V_{a w}^{2}=u_{a w}^{2}+v_{a w}^{2}$ is the square of the apparent wind speed; $u_{a w}=u_{w}-u$ and $v_{a w}=$ $v_{w}-v$ are the components of the apparent wind velocity; $u_{w}=-V_{w} \cos \left(\psi_{w}-\psi\right)$ and $v_{w}=-V_{w} \sin \left(\psi_{w}-\psi\right)$ are the components of the real wind velocity; $V_{w}=V_{R}+v_{g}$ is the (real) wind speed; $\psi_{w}$ is the incoming wind direction, assumed to be constant; $A_{f t}$ and $A_{l t}$ are the front and lateral top side areas, respectively; $C_{x_{w}}, C_{y_{w}}$, and $C_{n_{w}}$ are the coefficients of the wind forces and moment computed for head, lateral, and bow quartering wind, respectively; $\gamma_{a w}:=\arctan \frac{v_{a w}}{u_{a w}}-\pi$ is the relative angle between the vessel bow and the main direction of the incoming apparent wind.

\section{DP REGULATOR}

The regulator is the kernel of the DP system; its modular frame is drawn in Fig. 7. The inputs are position and velocity errors and sensor signals, while outputs are the setpoints sent to each actuator via allocation force and thrust logics. 


\section{A. Controller}

The controller consists of three independent PDs, one per axis. To correct the mean offset error, the usual integrative term is replaced by the reconstruction of the environmental disturbances to be compensated. In detail, wind action is directly reconstructed through the on-board anemometer measurements as a feedforward, while the current and the sea actions are estimated by means of an average of the force and moment required by the PD controller, repeated at every fixed time interval $\Delta t$.

Defining the input errors as

$$
\begin{aligned}
& e_{x}:=x-x_{d} \\
& e_{y}:=y-y_{d} \\
& e_{\psi}:=\psi-\psi_{d}
\end{aligned}
$$

where the arrays $\left\{x_{d}, y_{d}, \psi_{d}\right\}$ represent the desired position and heading, and indicating by $\left\{X_{R}, Y_{R}, N_{R}\right\}$ the components (relative to the $\underline{b}$-basis) of the required force and moment, the control law can be written as

$$
\begin{aligned}
{\left[\begin{array}{c}
X_{R} \\
Y_{R} \\
N_{R}
\end{array}\right]=} & {\left[\begin{array}{ccc}
\cos \psi & \sin \psi & 0 \\
-\sin \psi & \cos \psi & 0 \\
0 & 0 & 1
\end{array}\right] } \\
& \cdot\left[\begin{array}{c}
K_{\mathrm{PX}} e_{x}+K_{\mathrm{DX}} \dot{e}_{x} \\
K_{\mathrm{PY}} e_{y}+K_{\mathrm{DY}} \dot{e}_{y} \\
K_{\mathrm{PX}} e_{\psi}+K_{D N} \dot{e}_{\psi}
\end{array}\right]+\left[\begin{array}{c}
\bar{N}_{\mathrm{PD}}+N_{\mathrm{WE}} \\
\bar{Y}_{\mathrm{PD}}+Y_{\mathrm{WE}} \\
\bar{X}_{\mathrm{PD}}+X_{\mathrm{WE}}
\end{array}\right]
\end{aligned}
$$

where the pairs $\left\{K_{\mathrm{PX}}, K_{\mathrm{DX}}\right\},\left\{K_{\mathrm{PY}}, K_{\mathrm{DY}}\right\}$, and $\left\{K_{\mathrm{PN}}, K_{\mathrm{DN}}\right\}$ are the coefficients of the PD controller for the longitudinal and lateral axes and for the moment, respectively; the array $\left\{\bar{X}_{\mathrm{PD}}, \bar{Y}_{\mathrm{PD}}, \bar{N}_{\mathrm{PD}}\right\}$ represents the mean values of the $\mathrm{PD}$ controller outputs in a certain time interval. The array $\left\{X_{\mathrm{WE}}, Y_{\mathrm{WE}}, N_{\mathrm{WE}}\right\}$ is the feedforward action; it is composed by the reconstruction of wind force and moment implemented through (15) when input are wind speed and the relative incoming wind direction detected on board by means of the anemometer.

\section{B. Allocation}

The FAL inputs are the required total force and moment components $X_{R}, Y_{R}$, and $N_{R}$ in the body-fixed basis, expressed as percentage of the maximum allowable ones

$$
\begin{aligned}
& t_{X}^{R}=100\left(\frac{X_{R}}{X_{\mathrm{MAX}}^{\mathrm{AV}, \mathrm{AD}}}\right) \\
& t_{Y}^{R}=100\left(\frac{Y_{R}}{Y_{\mathrm{MAX}}^{\mathrm{AV}, \mathrm{AD}}}\right) \\
& t_{N}^{R}=100\left(\frac{N_{R}}{N_{\mathrm{MAX}}^{\mathrm{AV}, \mathrm{AD}}}\right) .
\end{aligned}
$$

Maximum allowable force and moment are defined as follows:

$$
\begin{aligned}
X_{\mathrm{MAX}}^{\mathrm{AV}} & =2 T_{\mathrm{AV}}^{\mathrm{MAX}} \\
X_{\mathrm{MAX}}^{\mathrm{AD}} & =2 T_{\mathrm{AD}}^{\mathrm{MAX}} \\
Y_{\mathrm{MAX}} & =L_{R}^{\mathrm{MAX}}+T_{\mathrm{BT}}^{\mathrm{MAX}} \\
N_{\mathrm{MAX}} & =L_{\mathrm{BP}} Y_{\mathrm{MAX}}
\end{aligned}
$$

where $L_{\mathrm{BP}}$ is the vessel length, $T_{\mathrm{AV}}^{\mathrm{MAX}}$ and $T_{\mathrm{AD}}^{\mathrm{MAX}}$ are the maximum forward and backward thrust of each screw propeller, respectively; $L_{R}^{\mathrm{MAX}}$ is the maximum lateral force deliverable by the rudder working downstream the propeller, and $T_{\mathrm{BT}}^{\mathrm{MAX}}$ is the maximum deliverable BT force. Because the vessel is not designed to maintain backward thrust continuously at the maximum rate, differences exist in the deliverable thrusts, in particular $T_{\mathrm{AV}}^{\mathrm{MAX}} \neq T_{\mathrm{AD}}^{\mathrm{MAX}}$

Finally, the allocation logic saturates forces to guarantee that $\left|t_{X}^{R}\right| \leq 100,\left|t_{Y}^{R}\right| \leq 100$, and $\left|t_{N}^{R}\right| \leq 100$.

To take measurement errors into account, dead zones have been introduced according to

$$
\begin{aligned}
& \varepsilon_{X} \leq\left|t_{X}^{R}\right| \leq 100 \\
& \varepsilon_{Y} \leq\left|t_{Y}^{R}\right| \leq 100 \\
& \varepsilon_{N} \leq\left|t_{N}^{R}\right| \leq 100
\end{aligned}
$$

where $\varepsilon_{X}=\varepsilon_{Y}=\varepsilon_{N}=5$.

The whole allocation logic envisages adopting the rudders (uncoupled) to generate lateral forces. In particular, the allocation algorithm requires the utilization of one rudder (called the active rudder or DP rudder), while the other is kept fixed hard over.

Once the FAL normalizes the required forces and moment, it is necessary to allocate the thrusts to each actuator via an appropriate TAL. In this regard, the adopted TAL criteria are as follows.

1) The propeller corresponding to the DP rudder has to be always in forward running, due to the low performances of the rudder when downstream of a reverse running propeller.

2) The second rudder is kept fixed at zero angle and the related propeller can deliver alternatively backward or forward force only.

3) The choice of the DP rudder is based on the sign of the scalar component $N_{R}(0)$ of the required moment at the initial instant of the DP maneuver, to ensure that, at least initially, the torque generated by the propellers is consistent with the required one. More precisely, we have:

a) the DP rudder is the portside one, if $N_{R}(0) \geq 0$;

b) the DP rudder is the starboard one, if $N_{R}(0)<0$.

The prerequisite of the TAL is the knowledge of the DP rudder angle, computed on the basis of the required force. Indeed, once the DP rudder angle is fixed, the remaining actuation unknowns reduce to the three thrusts, one for each corresponding thruster. Such thrusts are determined algebraically by the balance of forces and moments. 


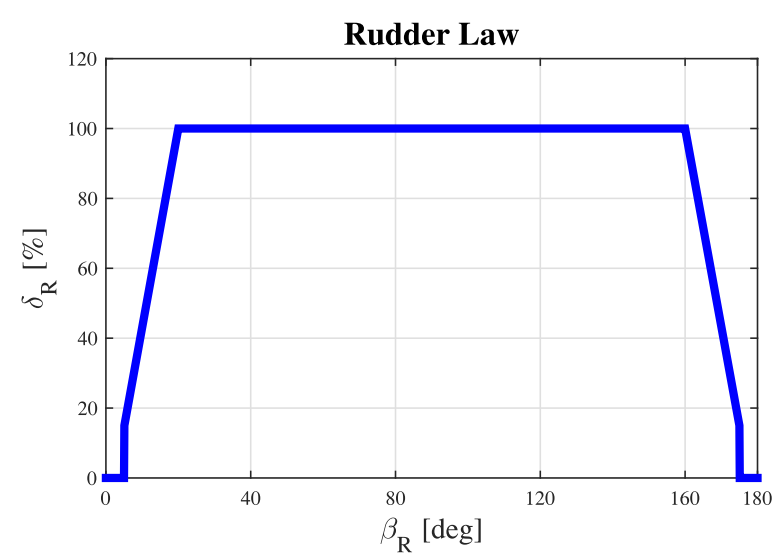

Fig. 8. Rudder angle choice law.

More in detail, longitudinal and lateral forces generated by the interaction between propeller and rudder are expressed according to the linear relations

$$
\begin{aligned}
& X=k_{D}\left(\delta_{R}\right) T \\
& Y=k_{L}\left(\delta_{R}\right) T
\end{aligned}
$$

where $k_{L}\left(\delta_{R}\right)=a_{L 1} \delta_{R}^{2}+a_{L 2} \delta_{R}+a_{L 3} \quad$ and $\quad k_{D}\left(\delta_{R}\right)=$ $a_{D 1} \delta_{R}^{2}+a_{D 2} \delta_{R}+a_{D 3}$ are the coefficients relating propeller thrust to rudder drag and lift forces, provided by the shipyard. As a result, the balance between required force and moment and the required thrusts is described by the relation

$$
\left[\begin{array}{c}
X_{R} \\
Y_{R} \\
N_{R}
\end{array}\right]=\mathbb{A}\left[\begin{array}{l}
T^{p t} \\
T^{s b} \\
T^{b t}
\end{array}\right]
$$

with

$$
\mathbb{A}=\left[\begin{array}{ccc}
k_{D}^{p t} & k_{D}^{s b} & 0 \\
k_{L}^{p t} & k_{L}^{s b} & 1 \\
x^{p t} k_{L}^{p t}-y^{p t} k_{D}^{p t} & x^{s b} k_{L}^{s b}-y^{s b} k_{D}^{s b} & x^{b t}
\end{array}\right]
$$

where $\left(x^{p t}, y^{p t}\right)$ and $\left(x^{s b}, y^{s b}\right)$ are the positions of the portside and starboard rudder hubs, which are assumed to be the thrust application points, and $\left(x^{b t}, 0\right)$ is the position of the BT hub.

As mentioned above, the crucial point is the evaluation of the DP rudder angle $\delta_{R}$. To this end, defining $\beta_{R}=\arctan \left(\frac{Y_{R}}{X_{R}}\right)$ for the four quadrants, up to the sign, the value of $\left|\delta_{R}\right|$ is determined according to the following law

$$
\left|\delta_{R}\right|= \begin{cases}0, & \left|\beta_{R}\right| \leq \beta_{d b} \\ \bar{\delta}+k\left|\beta_{R}\right|, & \beta_{d b}<\left|\beta_{R}\right| \leq \beta_{\text {ramp }} \\ \delta_{\text {MAX }}, & \beta_{\text {ramp }}<\left|\beta_{R}\right| \leq \pi\end{cases}
$$

where $\beta_{d b}$ is the dead-band angle; the introduction of this angle is necessary to avoid rudder activation for small $\beta_{R} ; \bar{\delta} \geq \delta_{\text {singular }}$ with $\delta_{\text {singular }}$ a critical angle making the allocation matrix singular, $\beta_{\text {ramp }}$ is the angle that determines the end of the ramp, and $k$ controls the ramp slop, as shown in Fig. 8. The introduction of $k$, as well as the dead-zone, reduces the machinery wearing parts and prevents the bang-bang effect.

The last step consists in choosing the sign of $\delta_{R}$. The sign is determined on the basis of the requirement that DP propeller is always forward running, according to the following procedure.
1) Case $N_{R}(0) \geq 0$

In such circumstance, the starboard rudder is hard over while the portside one is the DP one. Then, the portside propeller has to be forward running. Thus, the balance of force and moment yields the system of algebraic equations

$$
\left\{\begin{array}{l}
X_{R}=k_{D}^{p t} T_{R}^{p t}+k_{D}^{s b} T_{R}^{s b} \\
Y_{R}=T_{R}^{b t}+k_{L}^{p t} T_{R}^{p t} \\
N_{R}=x^{b t} T_{R}^{b t}+x^{p t} k_{L}^{p t} T_{R}^{p t}+ \\
\quad-y^{p t} k_{D}^{p t} T_{R}^{p t}-y^{s b} k_{D}^{s b} T_{R}^{s b}
\end{array}\right.
$$

From the system (24), the equation

$$
\begin{aligned}
N_{p t}^{*} & :=X^{p t}\left[k^{p t}\left(x^{b t}-x^{p t}\right)-y^{s b}+y^{p t}\right] \\
& =x^{b t} Y_{R}-y^{s b} X_{R}-N_{R}
\end{aligned}
$$

follows. The sign of $\left[k^{p t}\left(x^{b t}-x^{p t}\right)-y^{s b}+y^{p t}\right]$ is controlled by that of $k^{p t}$, which has the same sign of $\delta_{R}$. Since the right side of (25) is entirely determined by the output of the controller, the sign of $N_{p t}^{*}$ results to be known. Therefore, to ensure $T^{p t}>0$ the following criteria must be applied

$$
\left\{\begin{array}{l}
N_{p t}^{*}>0 \Rightarrow \delta_{R}>0 \\
N_{p t}^{*}<0 \Rightarrow \delta_{R}<0 .
\end{array}\right.
$$

2) Case $N_{R}(0)<0$

The portside rudder is hard over while the starboard one is the DP one. Then, the starboard propeller has to be forward running.

Applying the same considerations of the previous case, it is easily seen that the analogous of (25) is now given by

$$
\begin{aligned}
N_{s b}^{*} & :=X^{s b}\left[k^{s b}\left(x^{b t}-x^{p t}\right)-y^{s b}+y^{p t}\right] \\
& =x^{b t} Y_{R}+y^{p t} X_{R}-N_{R} .
\end{aligned}
$$

On the basis of (27), we have

$$
\left\{\begin{array}{l}
N_{s b}^{*}>0 \Rightarrow \delta_{R}>0 \\
N_{s b}^{*}<0 \Rightarrow \delta_{R}<0 .
\end{array}\right.
$$

Once $\delta_{R}$ is known, then the required thrusts are obtained by the inverse relation of (22). Of course, once the allocation procedure has singled out suitable thrusts for each propeller, corresponding control signals have to be sent to the propeller's local controllers. About this, the shaftline revolution setpoint is kept constant, with a value chosen by an intensive simulation campaign and resulting in a good compromise between performances and physical limits of the machineries (e.g., thrust-bearing not designed to operate in a bollard pull condition or minimum allowable shaftline revolution). Instead, the propeller pitch angle setpoint is generated by an interpolation of the relationships between thrusts and pitch angles assessed in steady-state conditions. The control of the BT follows the same strategy. 

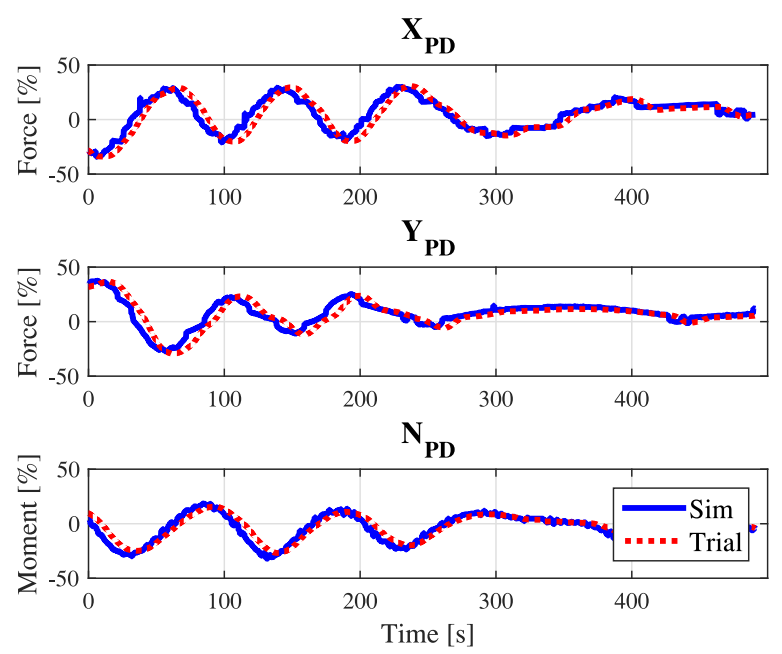

Fig. 9. PD controller outputs.

\section{Sea Trials and Simulation Results}

To validate the developed simulation platform, in this section comparisons between measurements recorded on board during sea trials and simulation results are presented. During the real maneuver, environmental conditions were characterized by Sea State 1 and wind of maximum mean speed of $6 \mathrm{kn}$, incoming from $200^{\circ}$ to $250^{\circ}$ with respect to the desired yaw angle $\psi_{d}$. The comparison analysis is carried out in two distinct steps: The first one concerns only the controller, while the second one involves the ship model in general, with particular attention devoted to the propulsion plant module.

It is worth noticing that it was not possible to detect and record some environmental disturbance characteristics, such as the time histories of the wave elevation and wave direction, as well as the current speed and current direction. For such a reason, the validation procedure is realized by means of open-loop simulation, where the position and velocity errors and the wind characteristics (magnitude and direction) sampled on board during sea trials are the inputs of the simulated controller instead of the outputs of the motion equations.

However, to verify the reliability of the whole simulation platform, also some closed-loop simulation results are presented and compared with sea trials data.

In the following figures, the dotted red lines stand for sea trials records, while the continuous blue line stands for simulation results. Moreover, forces are plotted as percentage of the maximum deliverable forces and moment, in accordance with (18), while motions are represented as percentage of the maximum allowable errors.

\section{A. Controller Validation}

In this section, the on-board and simulated controllers are compared: Input errors are the same for both controllers and coincide with position and velocity signals recorded on board; some relevant outputs are presented to test the control and allocation logics.
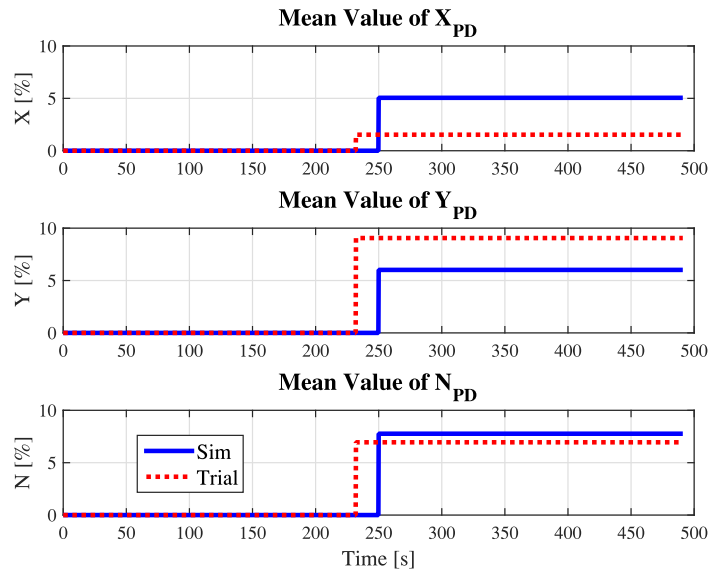

Fig. 10. Mean sea action estimation.
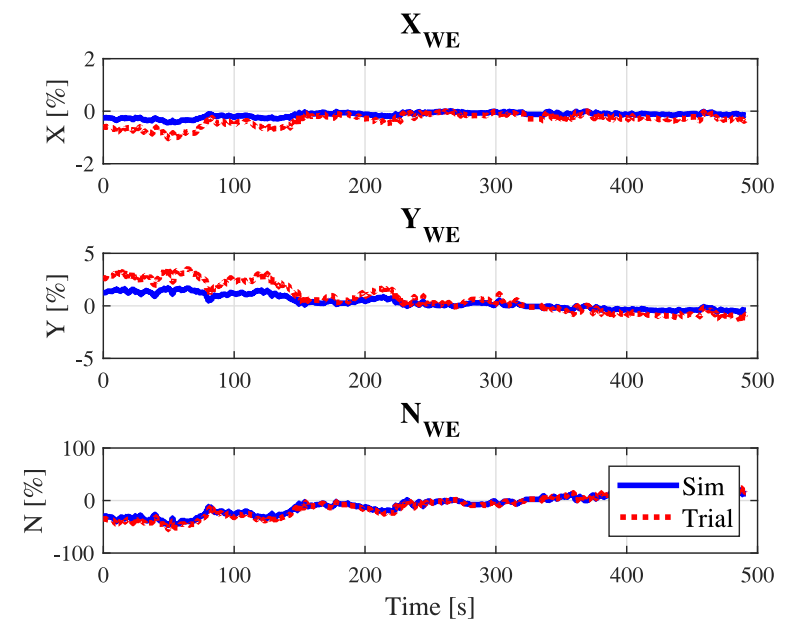

Fig. 11. Wind forces and moment reconstruction.

Fig. 9 presents PD-outputs of both true and simulated controllers. Small differences can be due to the signal delays detected on board. In the first part of the maneuver, errors are higher because the controllers are compensating only the wind action while computing the average of the sea action. As time passes, also sea loads are compensated and the control is smoother. Fig. 10 shows the mean of the wave and current forces estimated on board and during the simulation, namely those corresponding to $\left\{\bar{X}_{\mathrm{PD}}, \bar{Y}_{\mathrm{PD}}, \bar{N}_{\mathrm{PD}}\right\}$ in (17). Values are reported as percentage of $T_{\mathrm{AV}}^{\mathrm{MAX}}$. Such values are very close; some differences can be owed to the mean computation of quasi-zero mean signals. Fig. 11 shows the reconstruction of wind force and moment, corresponding to $\left\{X_{\mathrm{WE}}, Y_{\mathrm{WE}}, N_{\mathrm{WE}}\right\}$ in (17). Dotted red curves are the forces computed on board, while blue ones represent the wind forces simulated through the anemometer signals recorded on board.

Comparisons of the allocation algorithm are also reported in the next graphics. Fig. 12 shows the behavior of the angle $\beta_{R}$ defined in Section IV-B. Required thrusts relative to each actuator are compared in Fig. 13. In the figure, there are some large differences between the recorded and the simulated signals. This is probably due to the difference between the algorithms adopted 


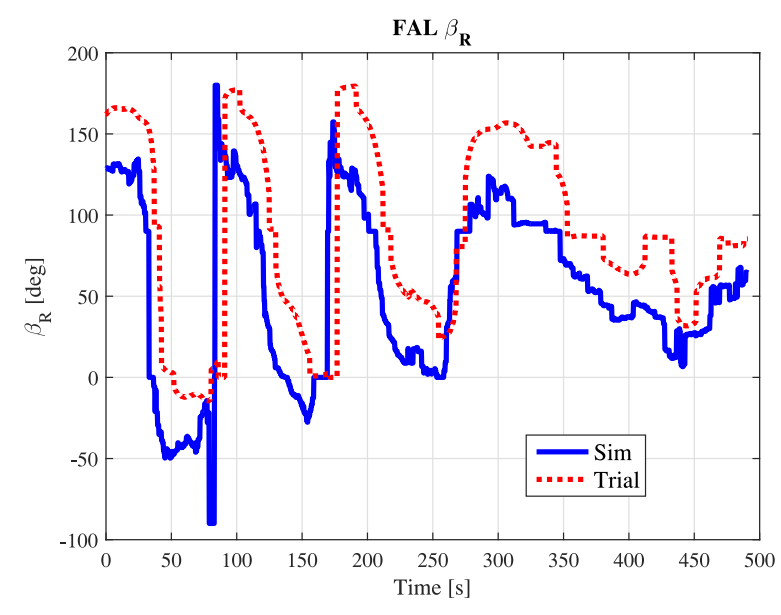

Fig. 12. FAL required angle.


Fig. 13. TAL required forces for each actuator.

on board and in the simulator for the computation of the inverse allocation matrix $\mathbb{A}^{-1}$, respectively.

Of course, the required thrusts have to be converted into shaft speed, pitch angle, and rudder angle requirements. Fig. 14 shows the required pitch angles; on the starboard side it is possible to note a little discrepancy between simulated and sea trials data. Fig. 15 shows the required rudder angles. Shaft speeds are kept constant, as it is illustrated in Fig. 16.

\section{B. Vessel Model Validation}

To validate the vessel model, first we focus on the propulsion plant. In this simulation phase, the inputs entering the model are the outputs of the controller obtained in the previous step, while the outputs are signals outgoing from the propulsion plant model.

Delivered pitch angles for both the propellers are plotted in Fig. 17, where differences in feedback time histories are the same as in the setpoint ones. It is seen that the model of propellers behaves correctly, as well as the rudder actuation model shown in Fig. 18.
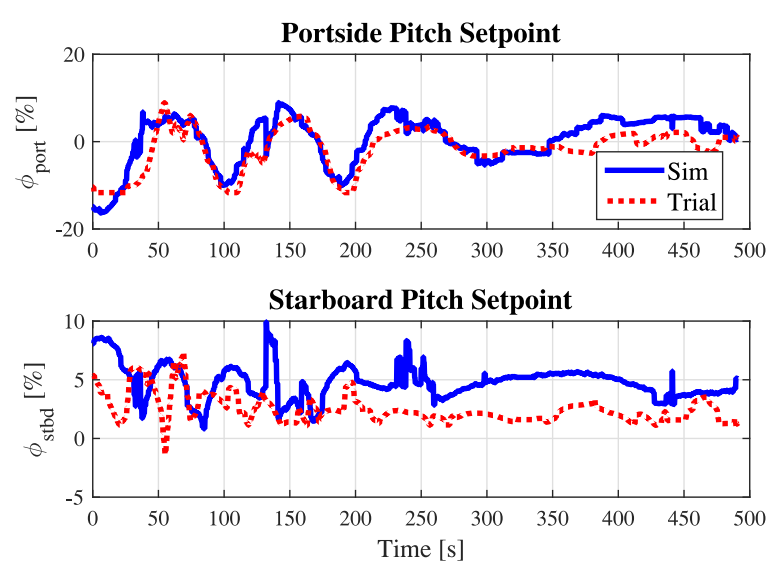

Fig. 14. TAL required pitch angle, values are percentage of their maximum.
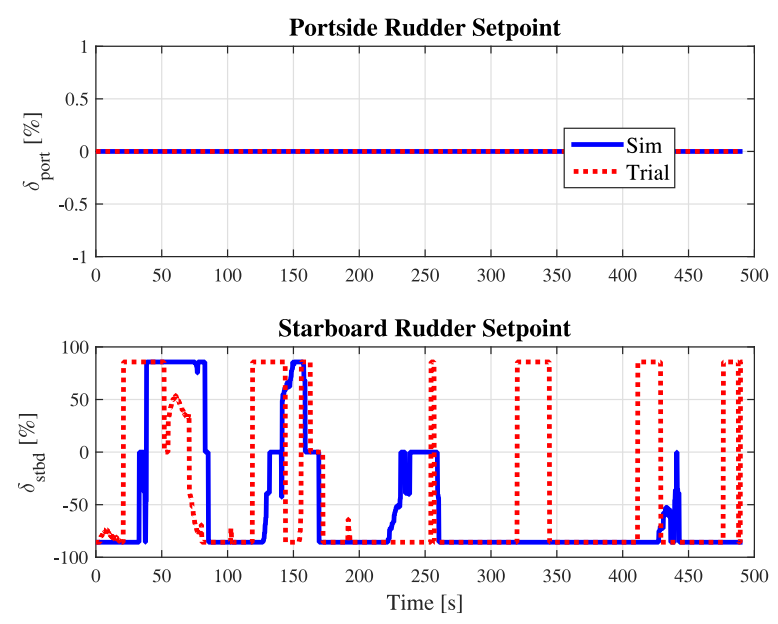

Fig. 15. TAL required rudder angle, values are percentage of their maximum
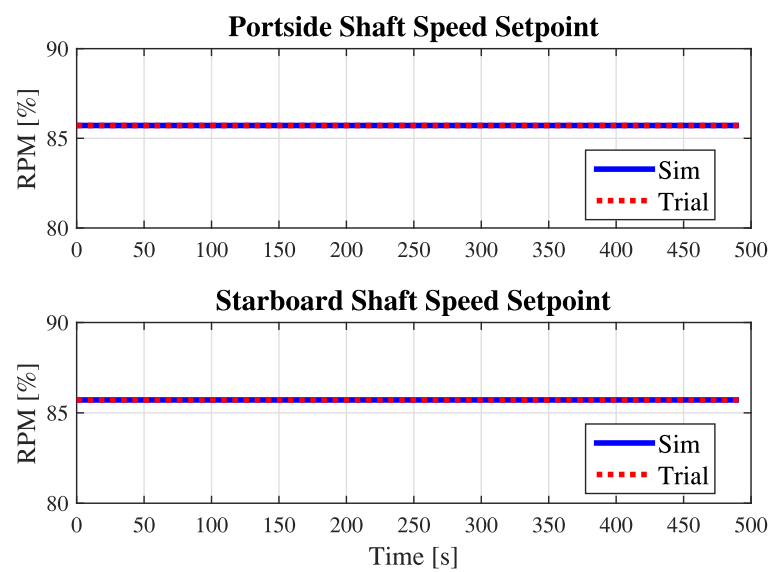

Fig. 16. TAL required shaft speeds, values are percentage of their maximum.

Figs. 19 and 21 contain data from the engine. In particular, Fig. 19 presents the time history of the delivered shaft speed, while the working points of the simulated engines are illustrated in the load diagrams (torque-revolution per minute) in Fig. 21. 

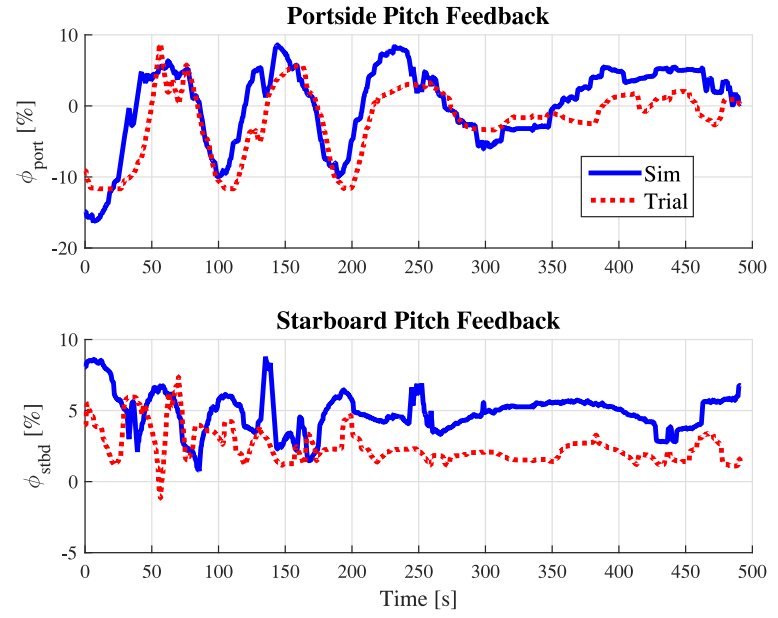

Fig. 17. Delivered pitch angles, values are percentage of their maximum.
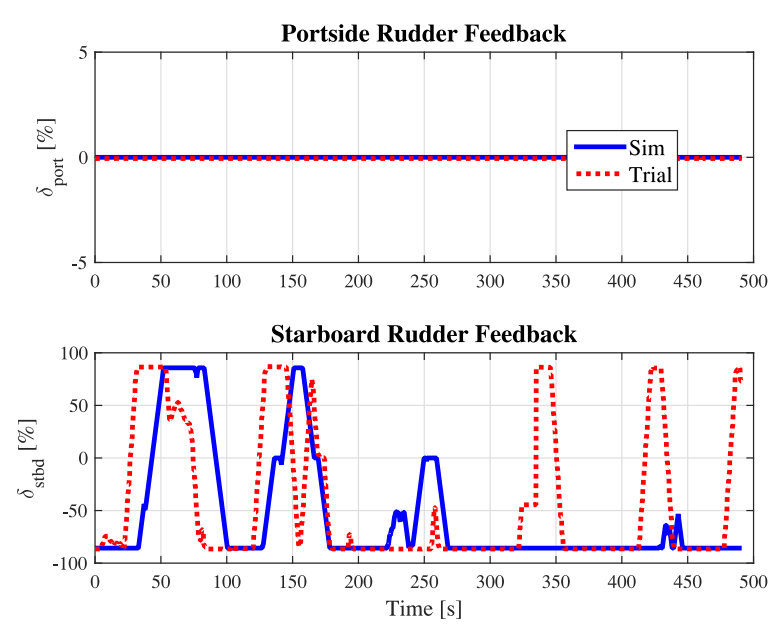

Fig. 18. Delivered rudder angles, values are percentage of their maximum.
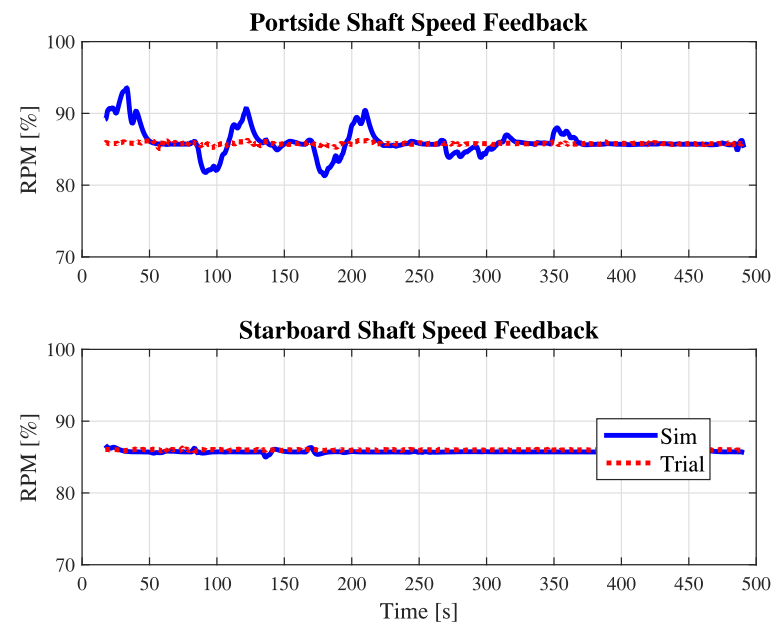

Fig. 19. Shaft speed, values are percentage of their maximum.

We notice that, due to technical reasons, the pitch angle setpoint for the BT could not be recorded on board.

Finally, a general overview of the DP maneuver entirely simulated in closed loop is illustrated and compared with sea trials
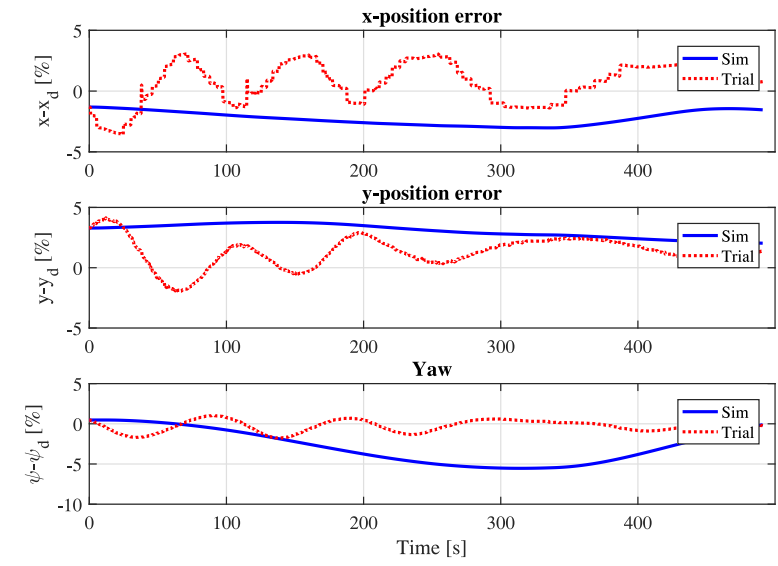

Fig. 20. Surge, sway, and yaw errors.
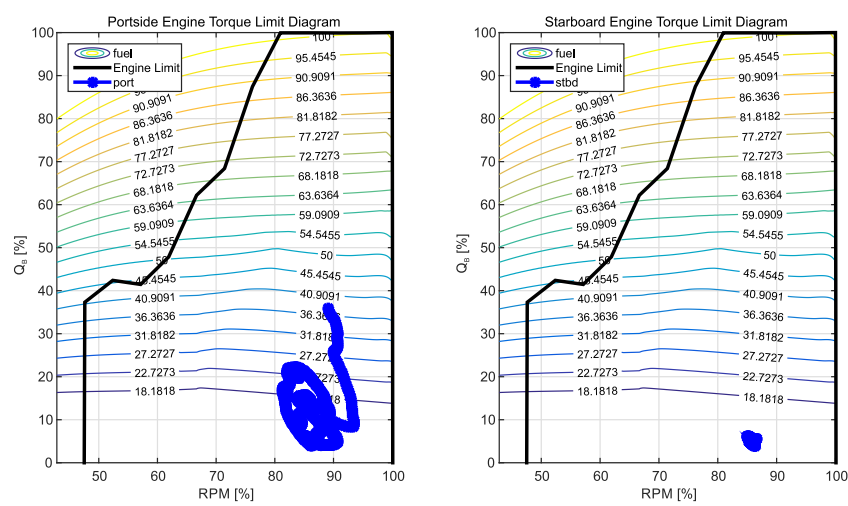

Fig. 21. Engine working points.
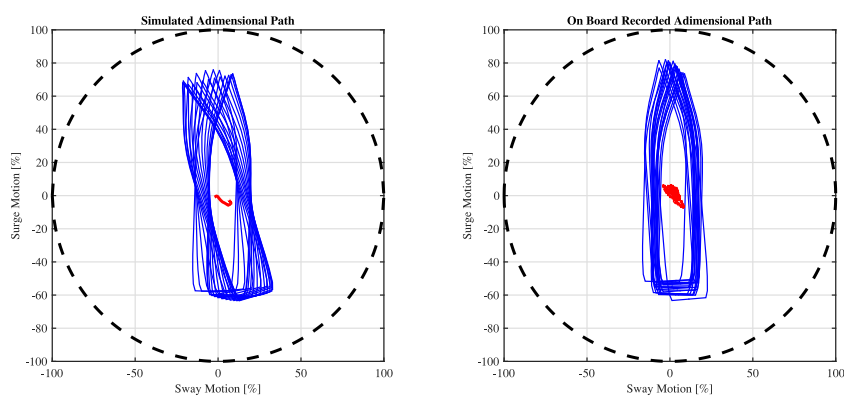

Fig. 22. Adimensional path.

data in Fig. 22. In this case, wind forces and moments are computed by means of the wind sensor recorded signals (incoming direction and wind speed time histories), while current and wave actions are simulated only by means of their mean forces and moments. For this reason, the presence of some discrepancies in Fig. 20 is mainly due to differences in the real and simulated disturbances time histories. Vessel position errors are reported in Fig. 22; the results show consistency between real and simulated motions, also in view of the fact that in the present case allowable errors in DP maneuvers are not very small as it happens in conventional DP applications. 


\section{CONCLUSION}

In this paper, the simulation methodology adopted to design and test the DP system for a vessel with a propulsion configuration not designed for DP purposes has been presented.

Simulation results and sea trial measurements have been compared to illustrate the reliability of the proposed simulation platform, as well as the effectiveness of the technical choices made both at control and allocation level. The unconventional propulsion configuration of the considered ship is one of the distinguishing and innovative aspects of the presented work. Indeed, DP control systems are usually designed for vessels specifically suited for DP tasks, where the kind and the number of the thrusters are suitably chosen to ensure high redundancy and efficiency in controlling the position and the orientation of the vessel. Nevertheless, through the simulation approach presented in this paper, it has been possible to show that even a ship with standard propulsion configuration can accomplish DP tasks in moderate weather conditions. The industrial interest in this field is evident, being possible to equip new as well as already existing conventional surface units of a DP system with good performances within certain limits of sea state. Another characteristic feature of the developed simulation approach is the presence of detailed propulsion and steering models able to represent the behavior of the ship plants with great accuracy. This is a fundamental aspect, not always properly addressed in other simulation works, to ensure fidelity of the simulation results and, in particular, to implement the RT-HIL procedure. Merging into a unique computational platform all the complex processes involved in the vessel motion makes possible to analyze the dynamic behavior of each single plant without losing sight of the overall performance of the ship. For instance, making use of the detailed propulsion plant model, suitable green control logics can be studied to reduce fuel consumption and/or exhaust emissions during DP maneuvers. A future work will be devoted to this topic.

\section{ACKNOWLEDGMENT}

The authors would like to thank SEASTEMA Spa for providing on board measurements data and for the provided scientific collaboration during the research project.

\section{REFERENCES}

[1] A. Alessandri et al., "Dynamic positioning system of a vessel with conventional propulsion configuration: Modeling and simulation," in Proc. Martech 2nd Int. Conf. Maritime Technol. Eng., 2014, pp. 725-733.

[2] M. Altosole, G. Benvenuto, M. Figari, and U. Campora, "Real-time simulation of a COGAG naval ship propulsion system,"J. Eng. Maritime Environ., vol. 223, no. 1, pp. 47-62, 2009.

[3] J. G. Balchen, N. A. Jenssen, and S. Slid, "Dynamic positioning using Kalman filtering and optimal control theory," in Proc. IFAC/IFIP Symp. Autom. Offshore Oil Field Oper., 1976, pp. 183-186.

[4] S. Donnarumma, M. Martelli, and S. Vignolo, "Numerical models for ship dynamic positioning," in Proc. 4th Int. Conf. Comput. Methods Mar. Eng., Rome, Italy, 2015, pp. 1078-1088.

[5] H. Fay, Dynamic Positioning Systems, Principles, Design and Applications. Paris, France: Editions Technip, 2005, Number 2-7108-0580-4.

[6] T. I. Fossen, "Marine control system," Mar. Cybern., Trondheim, Norway, 2002.

[7] T. A. Johansen and T. I. Fossen, "Control allocation-a survey," Automatica, vol. 49, no. 5, pp. 1087-1103, 2013.
[8] T. A. Johansen and A. J. Sørensen, "Experiences with HIL simulator testing of power management systems," Marine Technol. Soc., Houston, TX, USA, 2009.

[9] M. Martelli, Marine Propulsion Simulation: Methods and Results. Berlin, Germany: Walter de Gruyter, 2015.

[10] M. Martelli, M. Figari, M. Altosole, and S. Vignolo, "Controllable pitch propeller actuating mechanism, modeling and simulation," Proc. Inst. Mech. Eng., vol. 228, no. 1, pp. 29-43, 2014.

[11] M. Martelli, M. Viviani, M. Altosole, M. Figari, and S. Vignolo, "Numerical modelling of propulsion, control and ship motions in 6 degrees of freedom," Proc. Inst. Mech. Eng., vol. 228, no. 4, pp. 373-397, 2014.

[12] S. Michetti, M. Ratto, A. Spadoni, M. Figari, M. Altosole, and G. Marcilli, "Ship control system wide integration and the use of dynamic simulation techniques in the Fremm project," in Proc. Elect. Syst. Aircr. Railway Ship Propulsion, 2010, pp. 1-6.

[13] P. Oltmann and S. D. Sharma, "Simulation of combined engine and rudder maneuvers using an improved model of hull-propeller-rudder interactions," in Proc. 15th ONR Symp. Naval Hydrodyn., Sep. 1984.

[14] K. Y. Pettersen and T. I. Fossen, "Underactuated dynamic positioning of a ship-experimental results," IEEE Trans. Control Syst. Techn., vol. 8, no. 5, pp. 856-863, Sep. 2000.

[15] J. A. Pinkster, "Low frequency second order wave exciting forces on floating structures," Ph.D. dissertation, Delft Univ. Technol., Delft, The Netherlands, 1980

[16] O. J. Sørdalen, "Optimal thrust allocation for marine vessels," Control Eng. Pract., vol. 5, no. 9, pp. 1223-1231, 1997.

[17] A. J. Sørensen, "Structural issues in the design and operation of marine control systems," Annu. Rev. Control, vol. 29, no. 1, pp. 125-149, 2005.

[18] A. J. Sørensen, "A survey of dynamic positioning control systems," Annu. Rev. Control, vol. 35, no. 1, pp. 123-136, 2011.

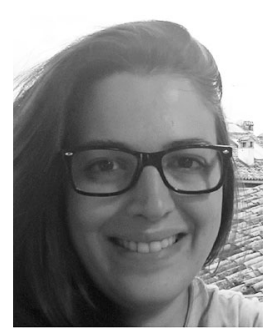

Silvia Donnarumma was born in Genoa, Italy, on December 21, 1986. She received the M.S. degree in naval architecture and marine engineering and the $\mathrm{Ph} . \mathrm{D}$. degree in mathematical engineering and simulation from the University of Genoa, Genoa, Italy, in 2012 and 2016, respectively.

In 2016, she had a research fellowship at the University of Genoa. Since 2017, she has been attending a postdoctoral research fellowship on automatic control at the University of Trento, Trento, Italy, mainly working on the study and application of nonlinear control techniques, based on hybrid approaches (with resets) and on convex optimization techniques based on LMIs, for the synthesis of feedback control systems. Her research interests include dynamic positioning system, control with actuator saturation, and automatic steering in general.

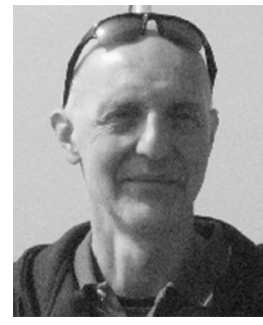

Massimo Figari received the M.S. degree in marine engineering and naval architecture from the University of Genoa, Genoa, Italy, in 1989.

From 1990 to 1991, he was a Marine Engineer in the Italian Navy. He was a Researcher at CETENA S.p.A., from 1991 to 1994, and in the Department of Naval Architecture and Marine Engineering, University of Genoa, from 1994 to 2002 . He has been an Associate Professor in the Politechnic School, University of Genoa, since 2002, and the Head of Master Course and Bachelor Course in Naval Architecture and Marine Engineering, since 2013. His teaching activities include military vessels, ship auxiliary systems, and reliability availability and safety of marine systems, and ship propulsion system (Italian Navy Academy, Livorno, Italy) His research interests include dynamic simulation of marine systems, reliability and safety of marine systems, environmental impacts of ships, and their consequence on the design. 




Michele Martelli was born in Italy in 1985. He received the B.S., M.S., and Ph.D. degrees in marine engineering and naval architecture from the University of Genoa, Genoa, Italy, in 2006, 2009, and 2013, respectively.

In 2015, he became a Research Fellow first and subsequently an Assistant Professor in the Department of Naval Architecture, Electric, Electronic, and Telecommunication Engineering, University of Genoa, Genoa, Italy, where he teaches an propulsion and auxiliary system course. He is a coauthor of 26 scientific papers. His main research interest focuses on the study of the dynamics of the propulsion plant and its control system. A second research topic regards the optimization of the propulsion efficiency. Both topics are mainly studied with simulation techniques. Since 2010 , he has been actively working on several research projects, funded by both private and public companies.

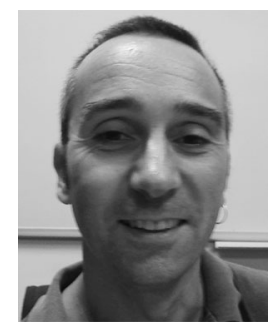

Stefano Vignolo was born in Genoa on February 2, 1972. He received the Laurea (summa cum laude) and $\mathrm{Ph} . \mathrm{D}$. degrees in mathematics from the University of Genoa, Genoa, Italy, in 1996 and 2001, respectively.

He was a Post-doctoral Fellow with a senior INDAM grant from 2001 to 2002 and a Research Fellow from 2002 to 2003 at the University of Genoa. He has been a Researcher with tenure in mathematical physics since 2004 and an Associate Professor in mathematical physics since 2014 at the University of Genoa. His research interests include classical mechanics, classical field theories, extended theories of gravity, mathematical models for control, and simulation in naval engineering.

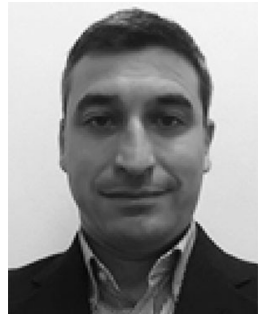

Michele Viviani received the M.S. degree in marine engineering and naval architecture from the University of Genoa, Genoa, Italy, in 2000.

$\mathrm{He}$ is currently an Associate Professor in the Department of Naval Architecture, Electric, Electronic, and Telecommunication Engineering, University of Genoa. His research interests include the areas of ship maneuverability (techniques for prediction and simulation of ship maneuverability characteristics, interaction between maneuverability and propulsion system, system identification), ship and leisure boats propulsors (experimental campaigns on conventional and nonconventional propellers and propulsors induced flow, cavitating behavior, induced pressures and radiated noise, propeller design with conventional methods and by means of multiobjective optimization), computational fluid dynamics, and hullform optimization. 